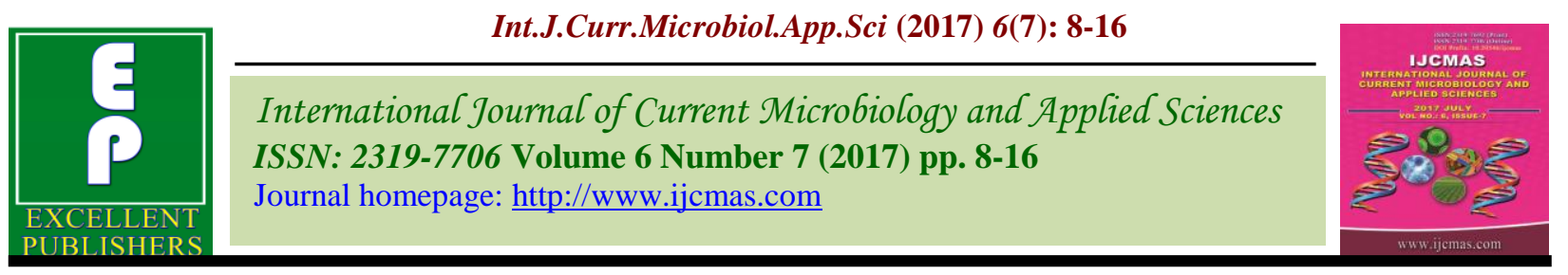

Original Research Article

https://doi.org/10.20546/ijcmas.2017.607.002

\title{
Gene Action and Combining Ability in Rice (Oryza sativa L.) Involving Indica and Tropical Japonica Genotypes
}

\author{
Ram Kishor ${ }^{1}$, Archana Devi' ${ }^{2}$ Preeti Kumari ${ }^{2}$, Saket Dwivedi $^{2}$, Ranjan Dwivedi', \\ S.P. Giri ${ }^{2}$, D.K. Dwivedi ${ }^{3 *}$ and U.P. Pandey ${ }^{4}$ \\ ${ }^{1}$ Indian Institute of Sugarcane Research, Lucknow (UP), India \\ ${ }^{2}$ Department of Genetics and Plant Breeding, N.D. University of Agriculture and Technology, \\ Kumarganj, Faizabad (UP), India \\ ${ }^{3}$ Department of Plant Molecular Biology and Genetic Engineering, \\ N.D. University of Agriculture and Technology, Kumarganj, Faizabad (UP), India \\ ${ }^{4}$ Department of Genetics and Plant Breeding, P. G. College, Ghazipur (UP), India \\ *Corresponding author
}

\begin{tabular}{|c|c|}
\hline \multicolumn{2}{|r|}{ A B S T R A C T } \\
\hline & \multirow{6}{*}{ 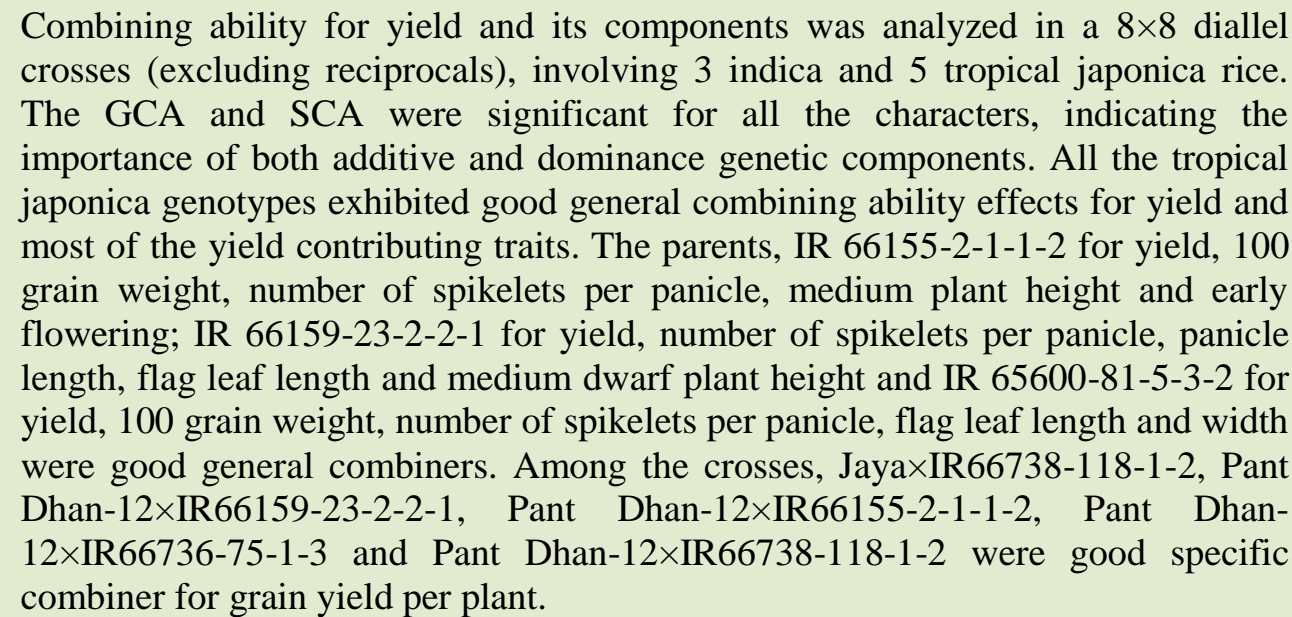 } \\
\hline Keywords & \\
\hline $\begin{array}{l}\text { Rice, } \\
\text { Combining ability, } \\
\text { Yield components. }\end{array}$ & \\
\hline Article Info & \\
\hline $\begin{array}{l}\text { Accepted: } \\
\text { 04 June } 2017 \\
\text { Available Online: } \\
\text { 10 July } 2017\end{array}$ & \\
\hline & \\
\hline
\end{tabular}

\section{Introduction}

The information on combining ability and gene action for different agronomic traits is important to achieve superior genotype from segregating population or in exploiting heterosis in rice. The combining ability of indigenous and tropical japonica in relation to enhance the magnitude of heterosis should be of immense value to breeder to use wide range of genetic basis for the improvement of yield and its components traits.
The combining ability analysis (Griffing, 1956) gives an idea about the relative magnitude of additive and non-additive type of gene action in expression of the traits.

Diallel analysis in rice has been reported by many workers (Shrivastava and Sheshu, 1983; Dwivedi et al., 1999; Munhot et al., 2000). The present investigation was made with a view to study the combining ability of 
indigenous and new plant type rice cultivars for yield and its component traits.

\section{Materials and Methods}

Eight divergent parents, three high yielding indica (Pant Dhan 12, Govind and Jaya) and five japonica lines (IR 65600-81-5-3-2, IR 66155-2-1-1-2, IR 66738-118-1-2, IR 6615923-2-2-1 and IR 66736-75-1-3) were selected for this study (Table 1). All possible, 28 crosses (excluding reciprocal) were made and part of the hybrid seeds were grown as off season crop to develop $F_{2}$ population and consisting of $28 \mathrm{~F}_{1} \mathrm{~s}, 28 \mathrm{~F}_{2} \mathrm{~s}$ and 8 parents. All the materials were grown in a Compact Family Randomized Block Design with three replications. Each plot of parents and $F_{1} s$ were a single row and that of $\mathrm{F}_{2} \mathrm{~s}$ had six rows each of 20 plants with the spacing of $20 \times 15$ $\mathrm{cm}$ row to row and plant to plant, respectively. Observations on days to $50 \%$ flowering, plant height, number of tillers per plant, flag leaf length, flag leaf width, panicle length, number of spikelets per panicle,100 grain weight and grain yield per plant were recorded on 15 randomly selected plants from parents and $\mathrm{F}_{1}$ and 50 plants from $\mathrm{F}_{2} \mathrm{~s}$, avoiding border plants. The combining ability analysis was carried out according to Griffing (1956) Model 1 Method 2.

\section{Results and Discussion}

Analysis of variance (Table 2) revealed that both general (GCA) and specific (SCA) combing ability to be highly significant for all the characters in both the generations (Table 1), indicating the involvement of both additive and non-additive type of gene action in expression of the characters. The relative magnitude of estimates of GCA variance was higher than those of SCA variance for all the characters, indicating the predominance additive gene action. The proportion of additive variance $\left(6^{2} \mathrm{~g}\right)$ was higher than non- additive genetic variance $\left(6^{2} \mathrm{~s}\right)$ for days to $50 \%$ flowering in both $\mathrm{F}_{1}$ and $\mathrm{F}_{2}$, revealing the importance of additive gene effects in the expression of the characters and conferring the earlier findings of Shrivastava and Sheshu, (1983) and Dwivedi et al., (1999). However, the non-additive gene action, as shown by higher estimates of $6^{2} \mathrm{~s}$ was found important for rest of the traits studies, similar to the findings reported by Dwivedi et al., (1999).

Non-additive component $\left(6^{2} \mathrm{~s}\right)$ was lower in magnitude in $F_{2}$ as compares to those of $F_{1}$ for number of tillers per plant, flag leaf length, flag leaf width, number of spikelets per panicle and grain yield. The decline of $6^{2} \mathrm{~s}$ in $\mathrm{F}_{2}$ may be expected due to increase in homozygosity and reduce in hererozygosity. The estimates of relative proportion of GCA and SCA $\left(26^{2} \mathrm{~g} / 26^{2} \mathrm{~g}+6^{2} \mathrm{~s}\right)$ as proposed by Baker (1978) were higher than 0.50 for characters, days to $50 \%$ flowering, flag leaf length, panicle length, number of spikelets per panicle and grain yield, indicating the role of additive gene action in expression of these characters. In view of the preponderance of additive genetic components in controlling these characters, it is expected that their genetic advance through single plant selection would be quite rewarding. Whereas, for other characters viz., plant height, number of tillers per plant, leaf width, and 100 grain weight, the ratio was lower than 0.50 , indicating the importance of non-additive gene action in governing the traits.

The estimates of GCA effects of parents (Table 3) showed that genotype IR 66155-21-1-2 was the best general combiner for yield and other yield contributing traits such as number of spikelets per panicle, 100 grain weight, early flowering, reduced plant height and short flag leaf followed by IR 66155-2-11-2, which exhibited good general combining ability for yield, reduced plant height, number 
of tillers per plant, long and narrow flag leaf, panicle length, number of spikelets per panicle and fine grain and IR 65600-81-5-3-2 showed good general combining ability for yield, number of spikelets per panicle and 100 grain weight. These tropical japonicas can be used to improve yield and its components because these lines were quite stable combiners which are evident from their high estimates of GCA over generations in diallel studies. Dwivedi and Pandey (2012) have also emphasized use of tropical japonicas having wide compatibility gene in rice improvement as well as for heterosis breeding.

The negative estimates of GCA effects are desirable for earliness and medium dwarf plant height. Among parents studied, Govind, IR 66155-2-1-1-2 and IR 66159-23-2-2-1 were proved good general combiners for earliness and short stature in both the generations. For more number of tillers Pant Dhan 12, Govind, Jaya and IR 66738-118-12; for short flag leaf Govind, Jaya, IR 661552-1-1-2 and IR 66736-75-1-3; for narrow leaf Govind, Jaya and IR 66159-23-2-2-1; for long panicle Pant Dhan 12, Jaya, IR 66738-118-12 and IR 66159-23-2-2-1; for number of spikeles per panicle, all tropical japonica genotypes; for bolder seed, IR 65600-81-5-32, IR 66155-2-1-1-2 and IR 66738-118-1-2 and other parents proved good general combiners for fine seeds. None of the parents were good general combiners for all the desirable traits studied.

The SCA effects and per se performance (Table 4) revealed that the cross combinations Jaya×IR66738-118-1-2, Jaya×IR66738-1181-2, Pant Dhan 12×IR66155-2-1-1-2, Pant Dhan 12×IR66736-75-1-3, Pant Dhan 12×IR66738-118-1-2, Govind $\times$ IR66738-1181-2, JayaxIR66736-75-1-3, Jaya×IR6615923-2-2-1, Govind $\times$ IR66155-2-1-1-2 and Govind $\times$ IR66159-23-2-2-1 were the superior specific combiners as they showed high positive significant SCA effects along with per se performance for yield and some of other yield components.

The cross combinations viz., JayaxIR66738118-1-2, Pant Dhan-12×IR66155-2-1-1-2, Pant Dhan-12×IR66736-75-1-3 and Pant Dhan-12×IR66738-118-1-2 exhibited positive and significant SCA effects for yield in both $F_{1}$ and $F_{2}$ generations. The additivex additive epistatic effects involved in these crosses could be fixed by simple breeding procedure.

Table.1 Origin and varietal type of rice genotypes used in the study

\begin{tabular}{|l|l|l|l|}
\hline S.No. & Varieties/Lines & Origin & Type \\
\hline 1. & Pant Dhan 12 & India & Indica \\
\hline 2. & Govind & India & Indica \\
\hline 3. & Jaya & India & Indica \\
\hline 4. & IR 65600-81-5-3-2 & IRRI, Philippines & Tropical Japonica \\
\hline 5. & IR 66155-2-1-1-2 & IRRI, Philippines & Tropical Japonica \\
\hline 6. & IR 66738-118-1-2 & IRRI, Philippines & Tropical Japonica \\
\hline 7. & IR 66159-23-2-2-1 & IRRI, Philippines & Tropical Japonica \\
\hline 8 & IR 66736-75-1-3 & IRRI, Philippines & Tropical Japonica \\
\hline
\end{tabular}




\section{Int.J.Curr.Microbiol.App.Sci (2017) 6(7): 8-16}

Table.2 Analysis of variance for general (gca) and specific (sca) combining ability for nine characters in rice

\begin{tabular}{|c|c|c|c|c|c|c|c|c|c|c|c|c|}
\hline S.No & Source & Generation & D.F. & $\begin{array}{l}\text { Days to } 50 \% \\
\text { flowering }\end{array}$ & $\begin{array}{l}\text { Plant } \\
\text { height }\end{array}$ & $\begin{array}{l}\text { No. of } \\
\text { tiller/plant }\end{array}$ & $\begin{array}{l}\text { Leaf } \\
\text { length }\end{array}$ & Leaf width & $\begin{array}{l}\text { Panicle } \\
\text { length }\end{array}$ & $\begin{array}{l}\text { No. of } \\
\text { spikelets/ } \\
\text { panicle }\end{array}$ & $\begin{array}{l}\text { 100- } \\
\text { grain } \\
\text { weight }\end{array}$ & $\begin{array}{l}\text { Grain } \\
\text { yield/plant }\end{array}$ \\
\hline \multirow[t]{2}{*}{1.} & \multirow[t]{2}{*}{ gca } & $\mathrm{F}_{1}$ & 7 & $275.84 * *$ & $40.19 * *$ & $28.63 * *$ & $19.08 * *$ & $0.05^{* *}$ & $4.42 * *$ & $2451.20 * *$ & $0.27 * *$ & $24.20 * *$ \\
\hline & & $\mathrm{F}_{2}$ & 7 & $280.85 * *$ & $37.30 * *$ & $28.32 * *$ & $15.73^{* *}$ & $0.04 * *$ & $4.48 * *$ & $2491.44 * *$ & $0.28 * *$ & $22.98 * *$ \\
\hline \multirow[t]{2}{*}{2.} & \multirow[t]{2}{*}{ sca } & $\mathrm{F}_{1}$ & 28 & $12.09 * *$ & $23.96 * *$ & $8.96 * *$ & $4.07 * *$ & $0.03 * *$ & $0.93 * *$ & $280.08 * *$ & $0.08 * *$ & $2.33 * *$ \\
\hline & & $\mathrm{F}_{2}$ & 28 & $13.60 * *$ & $24.08 * *$ & $7.98 * *$ & $2.70 * *$ & $0.01 * *$ & $1.12 * *$ & $269.73 * *$ & $0.09 * *$ & $2.20 * *$ \\
\hline \multirow[t]{8}{*}{3.} & \multirow[t]{2}{*}{ Error } & $\mathrm{F}_{1}$ & 70 & 1.22 & 1.45 & 0.51 & 0.79 & 0.008 & 0.47 & 2.48 & 0.02 & 0.16 \\
\hline & & $\mathrm{F}_{2}$ & 70 & 1.36 & 1.53 & 0.73 & 0.83 & 0.01 & 0.56 & 2.45 & 0.02 & 0.15 \\
\hline & $6^{2} g$ & $\mathrm{~F}_{1}$ & & 27.46 & 3.87 & 2.81 & 1.83 & 0.00 & 0.40 & 244.87 & 0.03 & 2.40 \\
\hline & & $\mathrm{F}_{2}$ & & 27.95 & 3.58 & 2.76 & 1.49 & 0.00 & 0.39 & 248.90 & 0.03 & 2.28 \\
\hline & $6^{2} s$ & $\mathrm{~F}_{1}$ & & 10.87 & 22.51 & 8.45 & 3.28 & 0.02 & 0.46 & 277.60 & 0.06 & 2.17 \\
\hline & & $\mathrm{F}_{2}$ & & 12.24 & 22.55 & 7.25 & 1.87 & 0.01 & 0.56 & 267.28 & 0.07 & 2.05 \\
\hline & $\begin{array}{l}26^{2} g / \\
\left(26^{2} g+6^{2} s\right)\end{array}$ & $\mathrm{F}_{1}$ & & 0.83 & 0.26 & 0.40 & 0.53 & 0.28 & 0.63 & 0.64 & 0.45 & 0.69 \\
\hline & & $\mathrm{F}_{2}$ & & 0.82 & 0.24 & 0.43 & 0.61 & 0.38 & 0.58 & 0.65 & 0.43 & 0.69 \\
\hline
\end{tabular}

$*$, ** Significant at $5 \%$ and $1 \%$ level, respectively 
Table.3 Estimate of genetic combining ability (gca) effects of $F_{1}$ and $F_{2}$ for different characters in 8 parent diallel crosses in rice

\begin{tabular}{|c|c|c|c|c|c|c|c|c|c|c|}
\hline \multirow[t]{2}{*}{ Parents } & \multicolumn{2}{|c|}{ Days to $50 \%$ flowering } & \multicolumn{2}{|c|}{ Plant height } & \multicolumn{2}{|c|}{ No. of tiller/plant } & \multicolumn{2}{|c|}{ Leaf length } & \multicolumn{2}{|c|}{ Leaf width } \\
\hline & $\mathrm{F}_{1}$ & $\mathrm{~F}_{2}$ & $\mathrm{~F}_{1}$ & $\mathrm{~F}_{2}$ & $\mathrm{~F}_{1}$ & $\mathrm{~F}_{2}$ & $\mathrm{~F}_{1}$ & $\mathrm{~F}_{2}$ & $\mathrm{~F}_{1}$ & $\mathrm{~F}_{2}$ \\
\hline Pant Dhan-12 & $0.26 * *$ & -0.05 & $3.25 * *$ & $2.73 * *$ & $0.49 * *$ & $0.79 * *$ & $0.22 * *$ & $0.18 * *$ & $-0.01 * *$ & 0.00 \\
\hline Govind & $-11.41 * *$ & $-11.62 * *$ & $-0.46^{* *}$ & -0.14 & $1.21 * *$ & $1.23^{* *}$ & $-1.01 * *$ & $-0.76^{* *}$ & $-0.07 * *$ & -0.06 \\
\hline Jaya & $6.09 * *$ & $5.98 * *$ & $1.01 * *$ & $0.59 * *$ & $2.14 * *$ & $2.13 * *$ & $-1.13 * *$ & $-1.5 * *$ & $-0.11 * *$ & $-0.09 * *$ \\
\hline IR 65600-81-5-3-2 & $1.66^{* *}$ & $2.08 * *$ & -0.02 & $0.01 * *$ & $-2.1 * *$ & $-2.05 * *$ & $0.94 * *$ & $0.94 * *$ & $0.03 * *$ & $0.04 * *$ \\
\hline IR 66155-2-1-1-2 & $-2.84 * *$ & $-2.42 * *$ & $-2.61 * *$ & $-2.97 * *$ & $-1.71 * *$ & $-1.89 * *$ & $-1.19 * *$ & $-1.24 * *$ & $0.02 * *$ & $0.02 * *$ \\
\hline IR 66738-118-1-2 & $2.46^{* *}$ & $2.18 * *$ & $2.05^{* *}$ & $2.28 * *$ & $1.73^{* *}$ & $1.49 * *$ & $0.62 * *$ & $0.63 * *$ & $0.01 * *$ & $-0.01 * *$ \\
\hline IR 66159-23-2-2-1 & $2.82 * *$ & $3.25 * *$ & $-1.32 * *$ & $-0.62 * *$ & $0.09 * *$ & 0.07 & $2.65 * *$ & $2.26 * *$ & $-0.01 * *$ & $-0.01 * *$ \\
\hline IR 66736-75-1-3 & $0.96 * *$ & $0.58 * *$ & $-1.9 * *$ & $-1.97 * *$ & $-1.85 * *$ & $-1.77 * *$ & $-0.91 * *$ & $-0.51 * *$ & $0.14 * *$ & $0.12 * *$ \\
\hline SE(gi) & 0.09 & 0.11 & 0.14 & 0.13 & 0.06 & 0.06 & 0.08 & 0.07 & 0.001 & 0.001 \\
\hline SE(gi-gi) & 0.22 & 0.27 & 0.32 & 0.31 & 0.14 & 0.14 & 0.19 & 0.17 & 0.002 & 0.002 \\
\hline $5 \%$ & 0.18 & 0.22 & 0.27 & 0.25 & 0.12 & 0.12 & 0.16 & 0.14 & 0.001 & 0.001 \\
\hline $1 \%$ & 0.23 & 0.28 & 0.35 & 0.33 & 0.15 & 0.15 & 0.21 & 0.18 & 0.0018 & 0.0023 \\
\hline
\end{tabular}

Table.3 Contd.

\begin{tabular}{|c|c|c|c|c|c|c|c|c|}
\hline \multirow[t]{2}{*}{ Parents } & \multicolumn{2}{|c|}{ Panicle length } & \multicolumn{2}{|c|}{ No. of spikelets/panicle } & \multicolumn{2}{|c|}{100 grain weight } & \multicolumn{2}{|c|}{ Grain yield/ plant } \\
\hline & $\mathrm{F}_{1}$ & $\mathrm{~F}_{2}$ & $\mathrm{~F}_{1}$ & $\mathrm{~F}_{2}$ & $\mathrm{~F}_{1}$ & $\mathrm{~F}_{2}$ & $\mathrm{~F}_{1}$ & $\mathrm{~F}_{2}$ \\
\hline Pant Dhan-12 & $0.97 * *$ & $0.99 * *$ & $-9.55 * *$ & $-12.35 * *$ & $0.02 * *$ & 0.00 & $0.70 * *$ & $-0.78 * *$ \\
\hline Govind & -0.05 & -0.1 & $-31.56 * *$ & $-31.32 * *$ & $-0.29 * *$ & $-0.25 * *$ & $-3.25 * *$ & $-3.14 * *$ \\
\hline Jaya & $0.25 * *$ & $0.13 * *$ & $-8.07 * *$ & $-6.6 * *$ & $-0.01 * *$ & $0.01 * *$ & $-0.6 * *$ & $-0.58 * *$ \\
\hline IR 65600-81-5-3-2 & $-0.19 * *$ & $-0.17 * *$ & $3.21 * *$ & $5.07 * *$ & $0.27 * *$ & $0.28 * *$ & $0.73 * *$ & $0.68 * *$ \\
\hline IR 66155-2-1-1-2 & $-0.53 * *$ & $-0.36 * *$ & $10.81 * *$ & $10.16^{* *}$ & $0.10 * *$ & $0.14 * *$ & $1.58 * *$ & $1.60 * *$ \\
\hline IR 66738-118-1-2 & $0.46^{* *}$ & $0.3^{* *}$ & $14.19 * *$ & $14.46 * *$ & $0.06^{* *}$ & $0.6^{* *}$ & $0.10 * *$ & $0.10 * *$ \\
\hline IR 66159-23-2-2-1 & $0.3 * *$ & $0.49 * *$ & $13.22 * *$ & $13.21 * *$ & $-0.03 * *$ & $-0.07 * *$ & $1.14 * *$ & $1.09 * *$ \\
\hline IR 66736-75-1-3 & $-1.21 * *$ & $-1.28 * *$ & $7.75 * *$ & $7.38 * *$ & $-0.13 * *$ & $-0.17 * *$ & $0.05^{* *}$ & $1.03 * *$ \\
\hline SE(gi) & 0.04 & 0.05 & 0.23 & 0.21 & 0.001 & 0.001 & 0.015 & 0.013 \\
\hline $\mathrm{SE}$ (gi-gi) & 0.09 & 0.11 & 0.53 & 0.49 & 0.004 & 0.003 & 0.035 & 0.03 \\
\hline $5 \%$ & 0.08 & 0.09 & 0.45 & 0.41 & 0.003 & 0.0027 & 0.029 & 0.025 \\
\hline $1 \%$ & 0.1 & 0.13 & 0.59 & 0.54 & 0.0046 & 0.0036 & 0.039 & 0.033 \\
\hline
\end{tabular}

$*$, ** Significant at $5 \%$ and $1 \%$ level, respectively 
Table.4 Estimate of specific combining ability (sca) effects of various characters in 8 parent diallel crosses in rice

\begin{tabular}{|c|c|c|c|c|c|c|c|c|c|c|}
\hline \multirow[t]{2}{*}{ Crosses } & \multicolumn{2}{|c|}{$\begin{array}{c}\text { Days of } 50 \% \\
\text { flowering }\end{array}$} & \multicolumn{2}{|c|}{ Plant Height } & \multicolumn{2}{|c|}{ No. of tiller/plant } & \multicolumn{2}{|c|}{ Leaf length } & \multicolumn{2}{|c|}{ Leaf width } \\
\hline & $\mathrm{F}_{1}$ & $\mathrm{~F}_{2}$ & $\mathrm{~F}_{1}$ & $\mathrm{~F}_{2}$ & $\mathrm{~F}_{1}$ & $\mathrm{~F}_{2}$ & $\mathrm{~F}_{1}$ & $\mathrm{~F}_{2}$ & $\mathrm{~F}_{1}$ & $\mathrm{~F}_{2}$ \\
\hline Pant Dhan-12×Govind & -1.41 & -1.57 & $-10.06^{* *}$ & $-9.63^{* *}$ & 0.39 & 0.15 & $2.75^{* * *}$ & $1.80^{* *}$ & $0.04^{\text {*** }}$ & $0.06^{* *}$ \\
\hline Pant Dhan-12×Jaya & 0.75 & 0.83 & $-5.34^{* * *}$ & -4.81 & $6.77^{* * *}$ & $6.35^{* * *}$ & -0.69 & $-1.98^{* * *}$ & $-0.27^{* *}$ & $-0.07^{* *}$ \\
\hline Pant Dhan-12×IR65600-81-5-3-2 & -0.48 & 1.06 & 1.75 & -0.37 & -0.43 & 0.19 & -1.45 & -2.28 & $-0.11^{* * *}$ & $0.05^{* *}$ \\
\hline Pant Dhan-12×IR66155-2-1-1-2 & 0.02 & -0.77 & 2.08 & 1.11 & -0.63 & -0.13 & $1.57 *$ & 0.48 & $0.13 * *$ & $0.08 * *$ \\
\hline Pant Dhan-12×IR66738-118-1-2 & $-1.95 *$ & -0.37 & 2.09 & 2.10 & 0.52 & 0.71 & $-2.53 * *$ & $-1.66^{*}$ & $0.15 * *$ & $0.03 * *$ \\
\hline Pant Dhan-12×IR66159-23-2-2-1 & 0.35 & 1.89 & $5.38 * *$ & $5.03 * *$ & 0.21 & -0.01 & -0.96 & -0.20 & $0.16 * *$ & $0.15 * *$ \\
\hline Pant Dhan-12×IR66736-75-1-3 & -0.45 & $-3.44 * *$ & $7.31 * *$ & $8.61 * *$ & $-2.32 * *$ & -1.10 & -0.47 & 0.15 & $0.08 * *$ & $0.06 * *$ \\
\hline Govind $\times$ Jaya & $-6.25^{* * *}$ & $6.27^{* *}$ & $-3.55^{* *}$ & $-3.21^{* * *}$ & $1.29^{* *}$ & 1.15 & 0.62 & $1.83^{* * *}$ & $0.08^{* *}$ & -0.02 \\
\hline Govind $\times$ IR65600-81-5-3-2 & $-3.81^{* *}$ & $-2.71^{* *}$ & $-3.47^{* *}$ & $4.14^{* *}$ & -1.08 & -0.72 & -1.01 & -1.09 & $-0.03^{* *}$ & -0.02 \\
\hline Govind $\times$ IR66155-2-1-1-2 & 0.69 & 0.79 & $2.90^{*}$ & $4.58^{* *}$ & 0.08 & 0.39 & $-1.97^{\text {*** }}$ & $-2.02^{* * *}$ & $0.14^{* *}$ & $0.14^{* * *}$ \\
\hline Govind $\times$ IR66738-118-1-2 & $-4.95^{* *}$ & $-5.47^{* * *}$ & 2.24 & 1.19 & $-1.64^{\text {*** }}$ & $-1.79^{* * *}$ & $-1.69^{* * *}$ & $-2.05^{* *}$ & $-0.03^{* *}$ & $-0.03^{* * *}$ \\
\hline Govind $\times$ IR66159-23-2-2-1 & $-2.31^{* *}$ & $-2.21^{*}$ & $3.89^{* * *}$ & $4.20^{* *}$ & 0.39 & 0.70 & $2.91^{* *}$ & $1.62^{*}$ & $0.17^{* * *}$ & $0.15^{\text {*** }}$ \\
\hline Govind $\times$ IR66736-75-1-3 & 0.45 & 0.13 & $4.72^{* * *}$ & $4.55^{* *}$ & $-2.66^{* *}$ & $-2.45^{* *}$ & $-1.60^{*}$ & 1.16 & $0.04^{* * *}$ & $0.01^{* * *}$ \\
\hline Jaya×IR65600-81-5-3-2 & $2.35^{* * *}$ & 2.03 & $5.28^{* * *}$ & $4.99^{* *}$ & $-4.65^{* *}$ & $-4.67^{* *}$ & 0.62 & 0.56 & -0.08 & $-0.04^{* * *}$ \\
\hline Jaya×IR65155-2-1-1-2 & $2.52^{* *}$ & $3.19^{* *}$ & $7.06^{* * *}$ & $5.15^{* *}$ & $-4.55^{* *}$ & $-4.33^{* *}$ & $-2.52^{* * *}$ & -1.11 & $0.20^{* *}$ & $0.21^{* * *}$ \\
\hline Jaya×IR66738-118-1-2 & -0.11 & 0.26 & 1.38 & 1.97 & $-4.32^{* * *}$ & $-4.04^{* * *}$ & 0.39 & -0.05 & $0.22^{* *}$ & $0.06^{* *}$ \\
\hline Jaya×IR66159-23-2-2-1 & -0.48 & -0.14 & 2.14 & 2.27 & $-5.31^{* *}$ & $-4.94^{* *}$ & $2.92^{* * *}$ & $2.49^{* *}$ & $0.16^{* *}$ & $0.13^{* *}$ \\
\hline Jaya×IR66736-75-1-3 & 1.39 & 1.86 & -0.36 & 0.51 & $-1.71^{* *}$ & $-1.59^{* *}$ & $3.15^{* *}$ & $2.32^{* *}$ & $0.29^{* *}$ & $0.26^{* *}$ \\
\hline IR65600-81-5-3-2× IR66155-2-1-1-2 & $-6.05 * *$ & $-4.91 * *$ & $9.55 * *$ & $-9.02 * *$ & 0.88 & 0.52 & -0.40 & 0.30 & $-0.09 * *$ & $-0.11 * *$ \\
\hline IR65600-81-5-3-2× IR66738-118-1-2 & 1.65 & 2.16 & 1.80 & 1.37 & $3.30 * *$ & $2.98 * *$ & 0.99 & 0.99 & $-0.10 * *$ & $-0.12 * *$ \\
\hline IR65600-81-5-3-2× IR66159-23-2-2-1 & 0.95 & -0.09 & -0.89 & $-3.26^{*}$ & $-1.18 * *$ & -0.46 & 0.87 & 0.06 & $0.02 *$ & $0.01 * *$ \\
\hline IR65600-81-5-3-2× IR66736-75-1-3 & $2.49 * *$ & $3.09 * *$ & $-4.84 * *$ & $-5.92 * *$ & 0.77 & 0.42 & $2.85 * *$ & $2.19 * *$ & $0.13^{* *}$ & $0.10 * *$ \\
\hline IR66155-2-1-1-2× IR66738-118-1-2 & $5.15 * *$ & $4.33 * *$ & $3.69 * *$ & $3.60 * *$ & $2.89 * *$ & $2.45 * *$ & 0.91 & 1.20 & $0.10 * *$ & $0.17 * *$ \\
\hline IR66155-2-1-1-2× IR66159-23-2-2-1 & $5.12 * *$ & $7.26^{*}$ & -0.12 & -1.15 & $-1.39 *$ & $-1.55 * *$ & $1.83 *$ & $1.57 *$ & $0.14 * *$ & $0.07 * *$ \\
\hline IR66155-2-1-1-2× IR66736-75-1-3 & 0.99 & $2.26 *$ & $5.51 * *$ & $6.75 * *$ & $1.38^{*}$ & 1.01 & 0.98 & 0.03 & $0.05 * *$ & $-0.02 * *$ \\
\hline IR66738-118-1-2× IR66159-23-2-2-1 & -0.18 & -0.34 & $-5.99 * *$ & $-4.07 * *$ & 0.02 & -0.36 & -0.32 & 0.45 & 0.00 & $0.05 * *$ \\
\hline IR66738-118-1-2× IR66736-75-1-3 & $3.35 * *$ & $3.33 * *$ & $-3.66 * *$ & $2.79 * *$ & 0.83 & 1.15 & -0.16 & 0.10 & $-0.22 * *$ & $-0.09 * *$ \\
\hline IR66159-23-2-2-1× IR66736-75-1-3 & $-2.35 * *$ & -1.74 & -0.21 & -1.57 & $3.91 * *$ & $3.63 * *$ & $-4.87 * *$ & $-4.03 * *$ & $0.02 * *$ & 0.00 \\
\hline \pm S.E (Sij) & 0.91 & \begin{tabular}{|l|}
1.12 \\
\end{tabular} & 1.32 & 1.26 & 0.58 & 0.60 & 0.77 & 0.69 & 0.006 & 0.009 \\
\hline \pm S.E (Sij-Sik) & 1.99 & 2.44 & 2.89 & 2.75 & 1.27 & 1.32 & 1.68 & 1.50 & 0.014 & 0.02 \\
\hline $5 \%$ & 1.78 & 2.20 & 2.59 & 2.47 & 1.14 & 1.18 & \begin{tabular}{|l|}
1.51 \\
\end{tabular} & 1.35 & 0.01 & 0.017 \\
\hline $1 \%$ & 2.34 & 2.88 & 3.39 & 3.24 & 1.49 & 1.54 & 1.98 & 1.17 & 0.02 & 0.023 \\
\hline
\end{tabular}


Table.4 Contd.

\begin{tabular}{|c|c|c|c|c|c|c|c|c|}
\hline \multirow[t]{2}{*}{ Crosses } & \multicolumn{2}{|c|}{ Panicle length } & \multicolumn{2}{|c|}{ No. of spikelets/panicle } & \multicolumn{2}{|c|}{100 grain weight } & \multicolumn{2}{|c|}{ Grain yield/ plant } \\
\hline & $\mathrm{F}_{1}$ & $\mathrm{~F}_{2}$ & $\mathrm{~F}_{1}$ & $\mathrm{~F}_{2}$ & $\mathrm{~F}_{1}$ & $\mathrm{~F}_{2}$ & $\mathrm{~F}_{1}$ & $\mathrm{~F}_{2}$ \\
\hline Pant Dhan- $12 \times$ Govind & 0.63 & 0.68 & $-13.65 * *$ & $-10.80 * *$ & -0.01 & $-0.03 * *$ & $-0.89 * *$ & $-0.57 * *$ \\
\hline Pant Dhan-12×Jaya & $-1.58 * *$ & $-1.33 * *$ & $-20.80 * *$ & $-21.58 * *$ & 0.01 & $-0.12 * *$ & $-0.86 * *$ & $-1.16^{* * *}$ \\
\hline Pant Dhan-12×IR65600-81-5-3-2 & 0.66 & 0.63 & $14.21 * *$ & -2.32 & $0.25 * *$ & $0.18 * *$ & 0.06 & $0.24 *$ \\
\hline Pant Dhan-12×IR66155-2-1-1-2 & 0.35 & 0.33 & $20.78 * *$ & $15.75 * *$ & $0.36^{* *}$ & $0.46 * *$ & $2.74 * *$ & $2.63 * *$ \\
\hline Pant Dhan-12×IR66738-118-1-2 & 0.33 & 0.17 & $16.78 * *$ & $20.62 * *$ & $-0.05^{* *}$ & $0.03 * *$ & $1.62 * *$ & $1.36^{* *}$ \\
\hline Pant Dhan-12×IR66159-23-2-2-1 & 0.09 & 0.67 & $22.64 * *$ & $24.20 * *$ & $0.42 * *$ & $0.53 * *$ & $2.77 * *$ & $2.62 * *$ \\
\hline Pant Dhan-12×IR66736-75-1-3 & 0.48 & 0.06 & $16.81 * *$ & $20.58 * *$ & $-0.18 * *$ & $-0.37 * *$ & $1.69 * *$ & $1.46^{* *}$ \\
\hline Govind $\times$ Jaya & 0.45 & 0.35 & $-14.54 * *$ & $-16.78 * *$ & $-0.26 * *$ & $-0.24 * *$ & $-2.02 * *$ & $-2.15 * *$ \\
\hline Govind $\times$ IR65600-81-5-3-2 & $1.55 * *$ & $1.56 * *$ & $4.36 * *$ & $9.03 * *$ & $0.23 * *$ & $0.16 * *$ & $-0.70 * *$ & $-0.46 * *$ \\
\hline Govind $\times$ IR66155-2-1-1-2 & $1.12 * *$ & 0.48 & -2.94 & -2.65 & $-0.43 * *$ & $-0.39 * *$ & $0.65 * *$ & $0.60 * *$ \\
\hline Govind $\times$ IR66738-118-1-2 & 0.58 & $-1.26^{*}$ & $10.48 * *$ & $10.21 * *$ & $0.64 * *$ & $0.68 * *$ & $1.26 * *$ & $1.19 * *$ \\
\hline Govind $\times$ IR66159-23-2-2-1 & 0.38 & -0.01 & -6.85 & $-6.05 * *$ & $-0.24 * *$ & $-0.21 * *$ & $0.36 *$ & $0.35 * *$ \\
\hline Govind $\times$ IR66736-75-1-3 & 0.05 & 0.5 & $27.59 * *$ & $23.65 * *$ & $0.17 * *$ & $0.21 * *$ & $0.42 * *$ & 0.17 \\
\hline Jaya×IR65600-81-5-3-2 & $0.95 * *$ & 0.5 & -0.46 & $22.25 * *$ & $-0.09 * *$ & $-0.13 * *$ & 0.11 & 0.16 \\
\hline Jaya×IR65155-2-1-1-2 & $0.97 * *$ & 0.65 & $22.27 * *$ & $19.90 * *$ & $-0.15^{* *}$ & $-0.15^{* *}$ & $0.42 * *$ & -0.03 \\
\hline JayaxIR66738-118-1-2 & $-0.81^{*}$ & -0.52 & $18.09 * *$ & $11.69 * *$ & 0.02 & $0.07 * *$ & $3.14 * *$ & $3.32 * *$ \\
\hline JayaxIR66159-23-2-2-1 & $0.74 *$ & -0.45 & $15.50 * *$ & $13.65 * *$ & $-0.09 * *$ & -0.03 & $0.76^{* *}$ & $0.95 * *$ \\
\hline Jaya×IR66736-75-1-3 & $1.33 * *$ & $1.61 * *$ & $26.12 * *$ & $23.92 * *$ & $0.49 * *$ & $0.58 * *$ & $0.83 * *$ & $0.94 * *$ \\
\hline IR65600-81-5-3-2× IR66155-2-1-1-2 & 0.20 & $1.24 * *$ & $-10.21 * *$ & $-9.78 * *$ & $0.04 *$ & $0.07 * *$ & $0.27 * *$ & 0.15 \\
\hline IR65600-81-5-3-2× IR66738-118-1-2 & 0.18 & -0.36 & -1.59 & -2.43 & $-0.09 * *$ & $-0.06 * *$ & $-1.07 * *$ & $-1.64 * *$ \\
\hline IR65600-81-5-3-2× IR66159-23-2-2-1 & -0.14 & $1.29 * *$ & 0.31 & -2.18 & $0.15 * *$ & $0.13 * *$ & $0.61 * *$ & 0.12 \\
\hline IR65600-81-5-3-2× IR66736-75-1-3 & $-0.88^{*}$ & $-2.09 * *$ & $-5.47 *$ & $-4.82 *$ & $-0.08^{*}$ & $0.02 *$ & $0.33 *$ & $-0.43 * *$ \\
\hline IR66155-2-1-1-2× IR66738-118-1-2 & -0.22 & 0.07 & $-11.38 *$ & $-7.17 * *$ & $-0.05 * *$ & $-0.03 * *$ & $-0.41 * *$ & $-0.37 * *$ \\
\hline IR66155-2-1-1-2× IR66159-23-2-2-1 & 0.41 & 0.26 & 0.15 & 0.44 & $0.21 * *$ & $0.15 * *$ & $0.57 * *$ & $-0.36 * *$ \\
\hline IR66155-2-1-1-2× IR66736-75-1-3 & $-1.65 * *$ & $-1.41 * *$ & $-12.60 * *$ & $-13.10 * *$ & $-0.34 * *$ & $-0.36^{* *}$ & $-1.14 * *$ & $-1.02 * *$ \\
\hline IR66738-118-1-2× IR66159-23-2-2-1 & -0.21 & -0.01 & $-9.10 * *$ & $-8.39 * *$ & $0.46^{* *}$ & $0.67 * *$ & $-1.23 * *$ & $-1.09 * *$ \\
\hline IR66738-118-1-2× IR66736-75-1-3 & $1.62 * *$ & $1.75 * *$ & -1.43 & -0.71 & $-0.04 *$ & $-0.11 * *$ & -0.11 & 0.05 \\
\hline IR66159-23-2-2-1× IR66736-75-1-3 & $-1.46^{* *}$ & $-1.03 * *$ & $-19.12 * *$ & $-18.18 * *$ & $-0.04 *$ & $-0.20 * *$ & $-0.72 * *$ & $-1.23 * *$ \\
\hline \pm S.E (Sij) & 0.36 & 0.46 & 2.18 & 2.02 & 0.02 & 0.01 & 0.15 & 0.12 \\
\hline \pm S.E (Sij-Sik) & 0.79 & 1.00 & 4.77 & 4.41 & 0.04 & 0.03 & 0.32 & 0.26 \\
\hline $5 \%$ & 0.71 & 0.90 & 4.27 & 3.96 & 0.04 & 0.02 & 0.29 & 0.24 \\
\hline $1 \%$ & 0.93 & 1.18 & 8.60 & 5.19 & 0.05 & 0.03 & 0.39 & 0.31 \\
\hline
\end{tabular}

$*$, ** Significant at $5 \%$ and $1 \%$ level, respectively. 
The cross combinations showing desirable SCA effects for days to $50 \%$ flowering (for earliness) were Govind $\times$ IR66738-118-1-2 and Govind $\times$ IR66159-23-2-2-1 in both generations, Pant Dhan-12 $\times$ IR66738-118-12 in $F_{1}$ and Pant Dhan-12 $\times$ IR66736-75-1-3 in $\mathrm{F}_{2}$ generation. For flag leaf length the negative estimates of SCA are desirable. The good specific combiners for short flag leaf were Pant Dhan $12 \times$ IR66738-118-1-2, Govind $\times$ IR66155-2-1-1-2 and Jaya $\times$ IR66159-23-2-2-1 (in F $\mathrm{F}_{1}$ ). For flag leaf width the negative estimates of SCA is desirable. The cross combination Govind $\times$ IR66738118-1-2 exhibited good specific combining ability for narrow flag leaf. For length of panicle the best specific combiners were Jaya $\times$ IR66736-75-1-3 in both the generations and Govind $\times$ IR66155-2-1-1-2 and Jaya $\times$ IR66159-23-2-2-1 in $\mathrm{F}_{1}$ generation only.

All the crosses exhibited good specific combing ability for number of spikelets per panicle except Govind × IR66159-23-2-2-1 and Govind $\times$ IR66155-2-1-1-2. The best specific combiners for bold seed size (100 grain weight) were Govind $\times$ IR66738-118-1 2 , Jaya $\times$ IR66736-75-1-3, Jaya $\times$ IR66738118-1-2 and Pant Dhan-12 $\times$ IR66155-2-1-1-2 and for fine seed size, Govind $\times$ IR66155-2-11-2, Govind $\times$ IR66159-23-2-2-1, Pant Dhan$12 \times$ IR66736-75-1-3, Jaya $\times$ IR66159-23-22-1 and Pant Dhan $12 \times$ IR66738-118-1-2 in $\mathrm{F}_{1}$ generation.

Further, the majority of the crosses, showed significant SCA effects which involved good and poor general combiners, indicating additive $x$ dominance type of gene interaction involved in the expression of characters. Few crosses Pant Dhan $12 \times$ IR66155-2-1-1-2, Pant Dhan $12 \times$ IR66155-2-1-1-2 and Pant Dhan $12 \times$ IR66155-2-1-1-2 having high $\times$ high general combiners showed high SCA effects and indicating the predominance of additive $\times$ additive type of gene effect.
Similar results have also been reported by Selvarani and Rangasamy, 1999, Salvaraj et al., 2011 and Dwivedi and Pandey (2012).

In case, where good $\times$ good general combiners are involved for high SCA effects, these crosses would be utilized for yield improvement through single plant selection in segregating generations. But in the crosses showing high SCA effects due to good $\times$ poor general combiners, simple pedigree breeding would not be effective to improve the characters. Population improvement i.e. mass selection with concurrent random mating in early segregating generations could be a perspective breeding procedure for yield improvement in rice. The crosses showing high SCA effects involving poor $\times$ poor general combiners could be exploited for heterosis breeding programme.

The GCA and SCA results indicated importance of both additive and dominance genetic components in the inheritance. All the tropical japonica genotypes exhibited good general combining ability effects for yield and most of the yield contributing traits. The tropical japonica lines having wide compatibility gene therefore exhibited fertile $\mathrm{F}_{1}$ hybrids with indica varieties. The parents, IR 66155-2-1-1-2 was good general combiner and cross Jaya $\times$ IR66738-118-1-2 was good specific combiner for grain yield per plant.

\section{References}

Baker, R.J., 1978. Issues in diallel analysis. Crop Science 18, 533-536.

Dwivedi, D.K., Pandey, M.P., Pandey, S.K., Rongbai, Li., 1999. Combining ability over environment in rice involving indica and tropical japonica lines. Oryza 36(2), 101-107.

Dwivedi, D.K., Pandey, M.P., 2012. Gene action and heterosis for yield and associated traits in indica and tropical 
japonica crosses of rice (Oryza sativa L.) involving wide compatibility gene(s). International Journal of Plant Breeding and Genetics 6(3), 140-150.

Griffing, B., 1956. Concept of general and specific combining ability in relation to diallel crossing system. Aust. J. Biol. Sci., 9, 463-493.

Munhot, M.K., Sarawagi, A.K., Rastogi, N.K., 2000. Gene action and combining ability for yield, grain quality and other related characters in rice. Oryza, 37, 16.

Selvaraj, C. I., Nagarajan, P., Thiyagarajan,
K., Bharathi, M., Rabindran, R., 2011. Studies on heterosis and combining ability of well-known blast resistant rice genotypes with high yielding varieties of rice (Oryza sativa L.) Int. J. Plant Breed. Genetics, 5, 111-129.

Selvarani, M., Rangasamy, P., 1999. Combining ability analysis in rice. Oryza, 36, 208-210.

Srivastava, M.N., Seshu, D.V., 1983. Combining ability for yield and its components in rice. Oryza, 32, 1-5.

\section{How to cite this article:}

Ram Kishor, Archana Devi, Preeti Kumari, Saket Dwivedi, Ranjan Dwivedi, S. P. Giri, D. K. Dwivedi and Pandey U. P. 2017. Gene Action and Combining Ability in Rice (Oryza Sativa L.) Involving Indica and Tropical Japonica Genotypes. Int.J.Curr.Microbiol.App.Sci. 6(7): 8-16. doi: https://doi.org/10.20546/ijcmas.2017.607.002 